\title{
ARTICLE PARP1 interacts with HMGB1 and promotes its nuclear export in pathological myocardial hypertrophy
}

\author{
Qian $\mathrm{LI}^{1,2,3}$, Zhuo-ming $\mathrm{LI}^{1,3}$, Shu-ya SUN ${ }^{1,3}$, Lu-ping WANG ${ }^{1,3}$, Pan-xia WANG ${ }^{1,3}$, Zhen GUO ${ }^{1,3}$, Han-wei YANG ${ }^{1,3}$, Jian-tao YE $^{1,3}$, \\ Jing $\operatorname{LU}^{1,2,3}$ and Pei-qing $\operatorname{LIU}^{1,2,3}$
}

High-mobility group box 1 (HMGB1) exhibits various functions according to its subcellular location, which is finely conditioned by diverse post-translational modifications, such as acetylation. The nuclear HMGB1 may prevent from cardiac hypertrophy, whereas its exogenous protein is proven to induce hypertrophic response. This present study sought to investigate the regulatory relationships between poly(ADP-ribose) polymerase 1 (PARP1) and HMGB1 in the process of pathological myocardial hypertrophy. Primary-cultured neonatal rat cardiomyocytes (NRCMs) were respectively incubated with three cardiac hypertrophic stimulants, including angiotensin II (Ang II), phenylephrine (PE), and isoproterenol (ISO), and cell surface area and the mRNA expression of hypertrophic biomarkers were measured. the catalytic activity of PARP1 was remarkably enhanced, meanwhile HMGB1 excluded from the nucleus. PARP1 overexpression by infecting with adenovirus PARP1 (Ad-PARP1) promoted the nuclear export of HMGB1, facilitated its secretion outside the cell, aggravated cardiomyocyte hypertrophy, which could be alleviated by HMGB1 overexpression. PE treatment led to the similar results, while that effect was widely depressed by PARP1 silencing or its specific inhibitor AG14361. Moreover, SD rats were intraperitoneally injected with 3-aminobenzamide (3AB, $20 \mathrm{mg} / \mathrm{kg}$ every day, a wellestablished PARP1 inhibitor) 7 days after abdominal aortic constriction (AAC) surgery for 6 weeks, echocardiography and morphometry of the hearts were measured. Pre-treatment of $3 A B$ relieved AAC-caused the translocation of nuclear HMGB1 protein, cardiac hypertrophy, and heart dysfunction. Our research offers a novel evidence that PARP1 combines with HMGB1 and accelerates its translocation from nucleus to cytoplasm, and the course finally causes cardiac hypertrophy.

Keywords: heart; HMGB1; PARP1; cardiac hypertrophy

Acta Pharmacologica Sinica (2019) 40:589-598; https://doi.org/10.1038/s41401-018-0044-4

\section{INTRODUCTION}

Myocardial hypertrophy, an adaptive response of the heart to multiple physiological or pathological stimulating factors, is featured by amplification of cardiomyocyte size, re-activation of fetal genes, and the recombination of cytoskeleton [1-3]. It is originally thought to be salutary but can evolve to heart failure (HF) if the stress continues [1]. Despite the progresses in the HF therapy, it is still one of the primary causes of death in most of the world [4]. Hence, illustration of the mechanisms underlying the development of myocardial hypertrophy is vital to explore efficient therapeutic tactics for the prevention and cure of HF.

High-mobility group box 1 (HMGB1) is a nuclear DNA-binding protein existing in diverse types of cells, including cardiomyocytes, which plays a part in preserving nucleosome structure, and regulates gene transcription, replication, and DNA repair [4-7]. Besides its nuclear activity, as an inflammatory cytokine, HMGB1 can be actively secreted by activated immune cells or passively released by necrotic cells [8-10]. It has been reported in the last few years that the nucleus expression of HMGB1 is obviously lower in human failing heart samples than that in normal hearts, due to the transposition from nucleus to cytoplasm in failing hearts [4].
The thoracic aorta constriction (TAC) induced the expression and translocation of HMGB1 in myocardium, and mechanical stress increased the expression and release of HMGB1 in cultured cardiomyocytes [11]. While exogenous HMGB1 protein is proven to induce hypertrophic response [12]. Moreover, the survival rate of HMGB1 transgenic mice were clearly higher than wild-type (WT) mice after TAC [4]. These findings suggest that nuclear HMGB1 is a negative regulator of myocardial hypertrophy.

Poly(ADP-ribose) polymerase 1 (PARP1), the foremost isoform of the PARP enzyme family, catalyzes the NAD+-dependent synthesis of the polymers of ADP-ribose (PAR) that consequently attach to target proteins, which is called poly(ADP-ribosyl)ation (PARylation) [13-16]. Throughout the years, much attention has been focused on the PARP1 hypotype, as it is responsible for producing most of PAR in the nucleus [17-22]. Previous study manifests that PARP $^{-1-}$ mice are protected against angiotensin II (Ang II)-stressed cardiac hypertrophy [23]. We reported that PARP1 is strongly activated by Ang II or isoproterenol (ISO), the PARylation of FoxO3 induced by PARP1 facilitates its phosphorylation at critical sites, leading to its translocation from the nucleus and finally resulting in cardiac hypertrophy $[24,25]$. In addition, it was reported that

\footnotetext{
${ }^{1}$ Department of Pharmacology and Toxicology, School of Pharmaceutical Sciences, Sun Yat-sen University, Guangzhou 510006 Guangdong, China; ${ }^{2}$ National and Local United

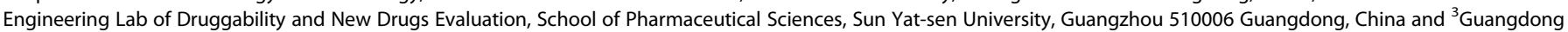
Provincial Key Laboratory of Construction Foundation, School of Pharmaceutical Sciences, Sun Yat-sen University, Guangzhou 510006 Guangdong, China Correspondence: Jing LU (lujing0504@126.com) or Pei-qing LIU (liupq@mail.sysu.edu.cn)
}

Received: 3 February 2018 Accepted: 10 May 2018

Published online: 20 July 2018 
PARP modified HMGB1 in vitro and influenced its subcellular relocalization during necrosis process [26].

In this study, HMGB1 was excluded from the nucleus after treatment with three hypertrophic stimulants (Ang II, phenylephrine (PE), or ISO) for $12 \mathrm{~h}$; PARP1 interacts with HMGB1 and promotes its nuclear export in cardiomyocytes; PARP1 silencing and its specific inhibitor AG14361 both retarded PE-induced HMGB1 nuclear export; the nuclear HMGB1 is partially responsible for the prevention of PARP1-mediated cardiac hypertrophy. We provide novel evidence that PARP1 combines with HMGB1 and accelerates its transposition from nucleus to cytoplasm, and finally causes cardiac hypertrophy.

\section{MATERIALS AND METHODS}

\section{Reagents}

Tyrisin for primary culture was purchased from Sigma-Aldrich (St. Louis, Missouri, USA). Ang II was purchased from EMD Millipore Corp (Billerica, MA USA). PE was bought from Tocris Bioscience (Bristol, UK). ISO was purchased from Sigma-Aldrich (St. Louis, Missouri, USA). AG14361 was obtained from Selleck Chemicals (Texas, USA). A total of 3-aminobenzamide (3AB) was achieved from MedChem Express (New Jersey, USA). Fetal Bovine Serum (FBS) was purchased from Cellmax (Beijing, China) and Thermo Fisher SCIENTIFIC (Massachusetts, USA). Dulbecco's modified Eagle's medium (DMEM) was obtained from Gibco (BRL Co. Ltd., USA). RIPA lysis buffer was obtained from Beyotime (Nantong, Jiangsu, China). The Nucleus Extract Kit was purchased from ACTIVE MOTIF (USA). Protease inhibitor cocktail was bought from Sigma-Aldrich (St. Louis, Missouri, USA). The enzyme linked immunosorbent assay (ELISA) kit was purchased from R\&D Systems (Minnesota, USA).

\section{Primary culture of neonatal rat cardiomyocytes}

Cardiomyocytes were isolated from the hearts of Sprague-Dawley (SD) rats (1-day-old to 3-day-old) as we previously described [27]. The myocardial cells were seeded at a density of $1 \times 10^{6}$ cells/well into 6-well plates in DMEM with $10 \%$ FBS and $100 \mu \mathrm{M}$ 5bromodeoxyuridine (5-BrdU). Ang II, PE, and ISO was respectively replenished to the final concentration of $1 \mu \mathrm{M}, 100 \mu \mathrm{M}, 10 \mu \mathrm{M}$, and the cells were sequentially incubated for referential time [27-29]. The same volume of DMEM was added as control group.

\section{Abdominal aortic constriction surgery, echocardiography, and morphometric measurement}

The animal experiments were conducted following the Guide for the Care and Use of Laboratory Animals (NIH Publication No. 8523, revised 1996) and were approved by the Research Ethics Committee of Sun Yat-Sen University. Male SD rats weighing 180$220 \mathrm{~g}$ (Certification No. 44005800005586, SPF grade) were from the Experimental Animal Center of Guangzhou University of Chinese Medicine (Guangzhou, China). A total of 36 rats were randomly assigned to Sham, $A A C$, or $A A C+3 A B$ group. As previously described [30], rats were injected with $10 \%$ chloral hydrate $(0.35 \mathrm{mg} / \mathrm{kg}$, ip) for anesthesia before surgery, under aseptic conditions, the abdominal aorta above the kidneys was exposed through a midline abdominal incision and constricted at the suprarenal level with a 6-0 silk suture tied twice around both the aorta and a blunted 22-gauge needle, the needle was then removed yielding a $70 \%-80 \%$ contraction. Sham-operated group underwent an analogical step without banding the aorta. $3 A B$ was injected to rats ( $20 \mathrm{mg} / \mathrm{kg}$ every day, ip) beginning from the next week of AAC surgery and lasting for 6 weeks.

Two-dimensional-guided M-mode echocardiography was accomplished by a Technos MPX ultrasound system (ESAOTE, SpAESAOTESpA, Italy) as formerly described [25]. After that, rats were immolated and then their hearts were quickly removed for trimming the left ventricles. Five-micrometer-thick histological cross sections of the heart tissues were stained with hematoxylin-eosin (HE) and Masson for morphometric measurement.

Quantitative real-time polymerase chain reaction

Total RNA was extracted from cultured neonatal rat cardiomyocytes (NRCMs) with Trizol reagent (Invitrogen, USA) and then was reversely transcripted to CDNA using Two-step RT Kit (Takara Biotechnology, Japan). The procedure included RT-A and RT-B steps. The mRNA levels of target genes were confirmed using SYBR-Green Quantitative PCR kit (Takara Biotechnology, Japan) by iCycler iQ system (Bio-Rad, USA). Amplification conditions were $95^{\circ} \mathrm{C}$ lasting $15 \mathrm{~min}$, followed by 40 cycles of $30 \mathrm{~s}$ at $95^{\circ} \mathrm{C}, 55^{\circ} \mathrm{C}$ for $1 \mathrm{~min}$, and $72^{\circ} \mathrm{C}$ for $30 \mathrm{~s}$. All polymerase chain reactions were conducted for five times. Rat-specific primers were synthesized by Sangon Biotech (Supplemental Table S1). $\beta$-actin served for an internal reference [31].

\section{Western blotting and co-immunoprecipitation analysis}

Total protein of cultured cells was extracted with RIPA lysis buffer containing a protease inhibitor cocktail on ice. Nucleus and cytoplasm proteins were extracted using Nucleus Extract Kit according to manufacturer's instruction.

For co-IP, anti-HMGB1 (diluted 1:50) was from Abcam (Cambridge, England), and anti-PARP1 (mouse, diluted 1:100) was obtained from Santa Cruz Biotechnology (Santa Cruz, CA, USA). The IP lysates $(400 \mu \mathrm{g})$ were incubated with the corresponding primary antibodies overnight (rabbit or goat normal lgG served as an control), and were incubated with protein $\mathrm{G}$-agarose beads (Pierce, Rockford, IL, USA) at $4{ }^{\circ} \mathrm{C}$ for $4 \mathrm{~h}$. The immunoprecipitated proteins were observed by Western blot analysis.

For Western blotting, primary antibody against PARP1 (mouse, diluted 1:500), was purchased from Santa Cruz Biotechnology (Santa Cruz, CA, USA). Primary antibody against HMGB1 (rabbit, diluted 1:10,000) was purchased from Abcam (Cambridge, England). Primary antibodies against a-Tubulin (rabbit, diluted 1:1000) and Lamin B1 (mouse, diluted 1:5000) were purchased from Proteintech Group, Inc (Chicago, USA). Antibody against PAR (mouse, diluted 1:500) was purchased from Trevigen (Gaithersburg, MD, USA). Western blot analysis was operated as previously described [32]. The Image J software (National Institutes of Health, USA) was used to analyse the intensity of protein bands.

Plasmids transfection and RNA interference

The plasmid pcDNA3.1-6Flag-HMGB1 was purchased from Genechem (Shanghai, China). Transient transfection of NRCMs was conducted using Lipofectamine LTX ${ }^{\circledR}$ \& PLUSTM reagent (Invitrogen, USA) on the basis of manufacturer's instruction. The successful overexpression of HMGB1 gene was determined by Western blot analysis (Supplemental Fig. S3). Small RNAs (siRNAs) for PARP1 and negative control were purchased from RiboBio (Guangzhou, China). The sequence was listed in Supplement Table S2. RNA interference was operated using Lipofectamine ${ }^{\circledR}$ RNAiMAX Reagent. The experiment protocol was on the basis of manufacturer's instrument. Western blot was carried out to verify silencing efficiency [33].

Measurement of cell surface area

NRCMs plated on 96 -well plate were fixed with $4 \%$ paraformaldehyde for $10 \mathrm{~min}$. Remove $4 \%$ paraformaldehyde and wash cells with phosphate buffer solution (PBS), then cells were permeabilized with $0.3 \%$ Triton $\mathrm{X}-100$ for $10 \mathrm{~min}$ at room temperature. Remove Triton and wash cells with PBS and use rhodaminephalloidin to dye for $30 \mathrm{~min}(50 \mu \mathrm{L} /$ well). Wash cells with PBS and stain the nucleus with DAPI ( $50 \mu \mathrm{L} /$ well) for 5 min away from light. Finally, wash cells and acquire images using High Content Screening system (Thermo Fisher Scientific, USA). Cell surface 
a
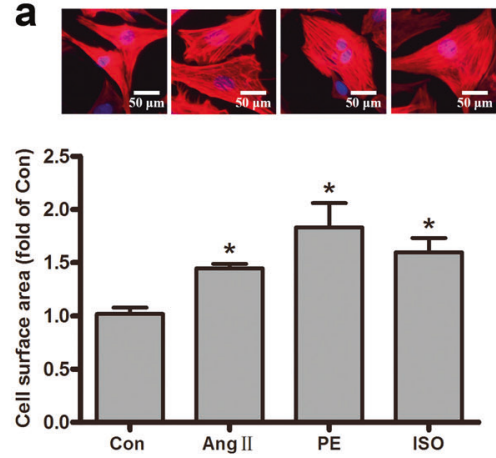

b

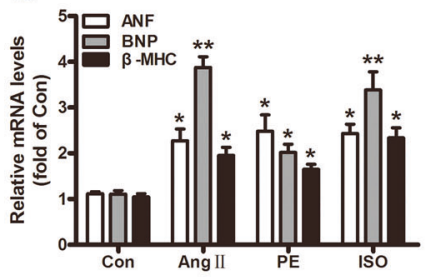

C

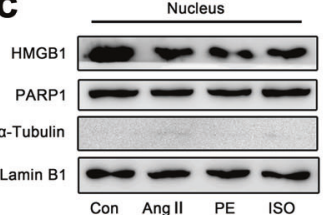

Cytoplasm
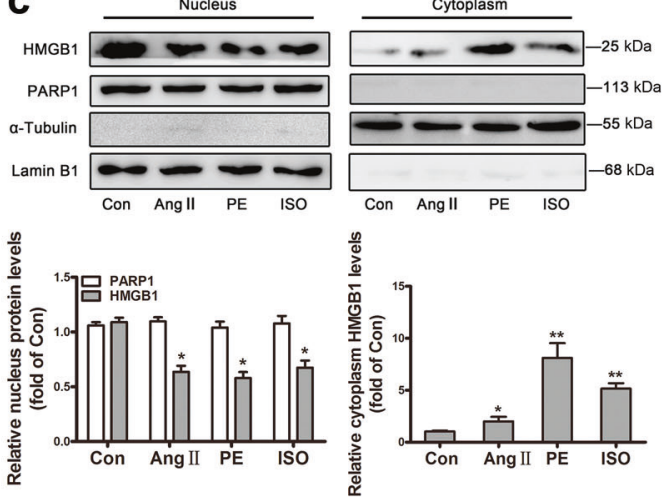
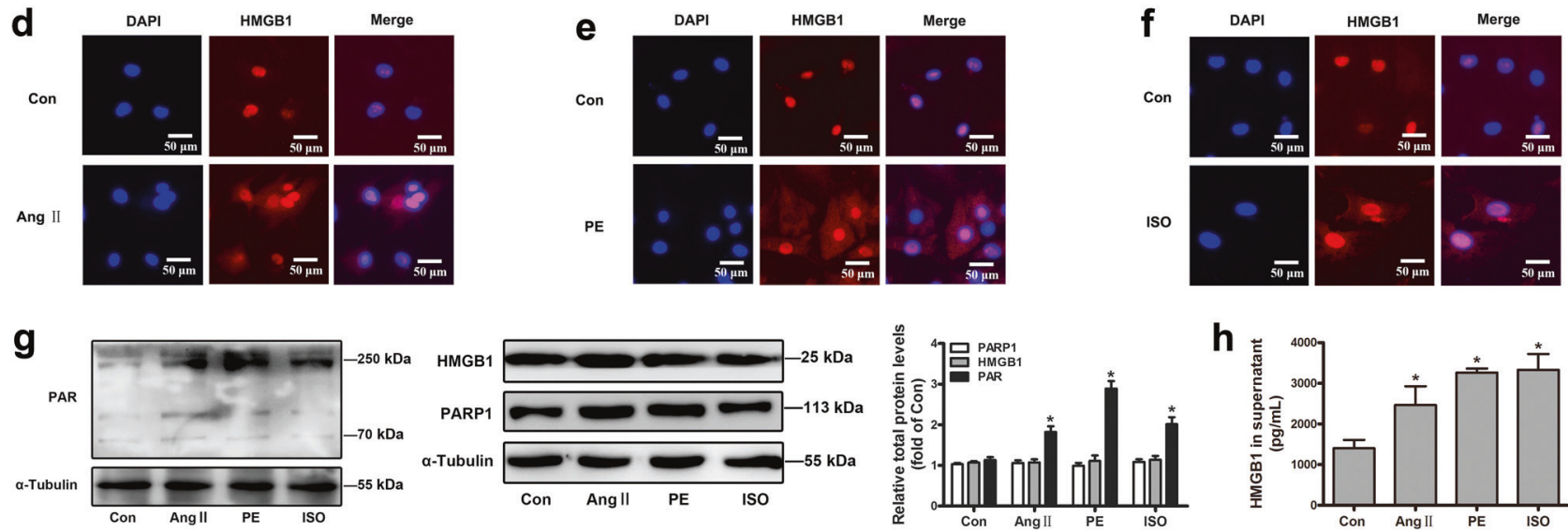

Fig. 1 Changes of PARP1 and HMGB1 in the hypertrophic cardiomyocytes. NRCMs were incubated with $1 \mu \mathrm{M}$ Ang II, $100 \mu \mathrm{M}$ PE, and $10 \mu \mathrm{M}$ ISO for the times indicated. a After $48 \mathrm{~h}$, the cell surface area was measured by dying with rhodamine-phalloidin in NRCMs. $\mathbf{b}$ After $12 \mathrm{~h}$, the mRNA levels of ANF, BNP, and $\beta$-MHC were confirmed using real-time PCR analysis. c After $12 \mathrm{~h}$, the nuclear and cytoplasmic protein were extracted respectively from NRCMs, and were observed by Western blot analysis. d-f After $12 \mathrm{~h}$, immunofluorescence (IF) assay was conducted to determine the subcellular distribution of HMGB1. Representative images of five independent experiments are shown. $\mathbf{g}$ After $24 \mathrm{~h}$, Western blot analysis was operated to confirm the intracellular protein of PAR, HMGB1, and PARP1. The results were normalized to those of $\alpha$-Tubulin/Lamin B1 and were presented as the means \pm SEM. $\mathbf{h}$ ELISA experiment was conducted to measure the level of HMGB1 in cell supernatant. ${ }^{*} P<0.05,{ }^{*} P<0.01$ vs control group, $n=5$. Con means control

area from randomly selective view (30 for each group) was measured with the build-in Image Analysis Software [28].

Immunofluorescence assay

NRCMs cultured on NEST 96-well plate were fixed with $4 \%$ paraformaldehyde. Then dry the cells in air for $10 \mathrm{~min}$. After washing three times with PBS, the cells were permeabilized with $0.5 \%$ Triton X-100 for $10 \mathrm{~min}$ and subsequently incubated at room temperature for $30 \mathrm{~min}$ in $10 \%$ goat serum. Afterwards, the cells were treated with primary antibody of HMGB1 or PARP1 overnight. Then the secondary antibody (diluted 1:200) was incubated with cells. Finally, the cells were tested using EVOS FL Auto (Life Technologies). The Image J software was used to quantify fluorescence intensity [34].

Enzyme linked immunosorbent assay

The concentration of HMGB1 in the cardiomyocytes supernatants was measured on the basis of the manufacturer's instruction. The absorbance at $\lambda / \mathrm{nm}=450$ was confirmed by Multiscan Spectrum (Molecular Devices, USA) microplate reader immediately in each sample well.

\section{Statistical analysis}

Data were presented as mean \pm standard error of the mean. Difference between two groups was analyzed by Student's $t$ test. Statistical analysis among multiple groups was performed by one- way ANOVA analysis. In all cases, a value of $P<0.05$ was considered statistically significant.

\section{RESULTS}

Changes of PARP1 and HMGB1 in the hypertrophic cardiomyocytes

In our study, three hypertrophic stimulants were respectively applied, including angiotensin II (Ang II, an active form of angiotensin), phenylephrine ( $P E$, a selective a1-adrenergic receptor agonist) and isoproterenol (ISO, a nonselective $\beta$-adrenergic receptor agonist) $[25,27]$. NRCMs were incubated with $1 \mu \mathrm{M}$ Ang II, $100 \mu \mathrm{M}$ PE and $10 \mu \mathrm{M}$ ISO for the times indicated [27-29]. All three of hypertrophic models displayed apparent cardiomyocyte hypertrophy according to the increased cell surface area and the mRNA levels of well-established hypertrophic biomarkers ANF (atrial natriuretic factor), BNP (brain natriuretic polypeptide), and $\beta-$ MHC ( $\beta$-myosin heavy chain) (Fig. $1 a, b)$.

To detect the changes of subcellular localization of HMGB1 in the process of cardiac hypertrophy, the nuclear and cytoplasmic protein were respectively extracted from NRCMs, and were observed by Western blot analysis. Besides, immunofluorescence (IF) assay was performed to determine the subcellular distribution of HMGB1. As shown in Fig. 1c, d-f, HMGB1 was mainly distributed in the nucleus rather than the cytoplasm of normal NRCMs, but excluded from the nucleus after treatment with three stimulants 
a
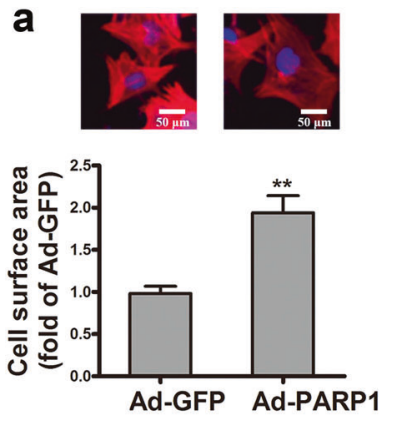

b
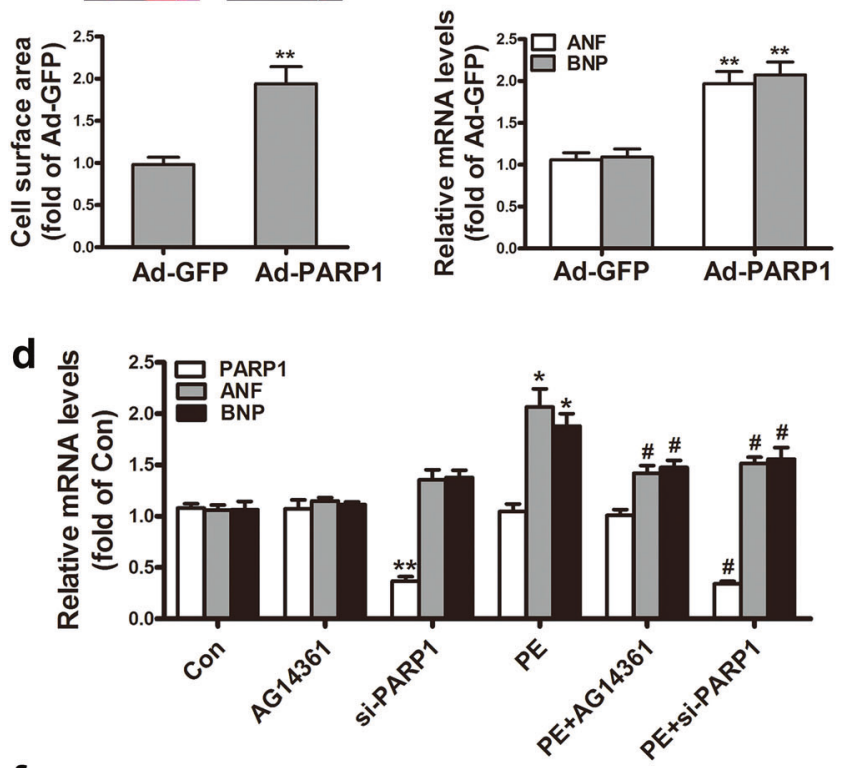

f

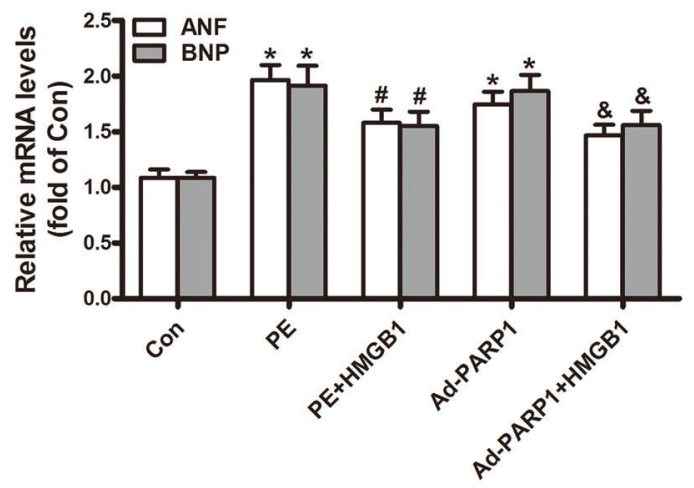

C

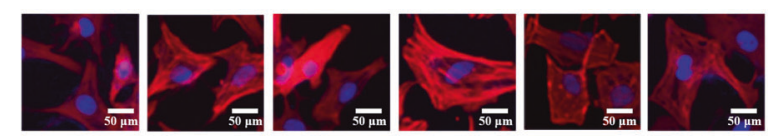

e
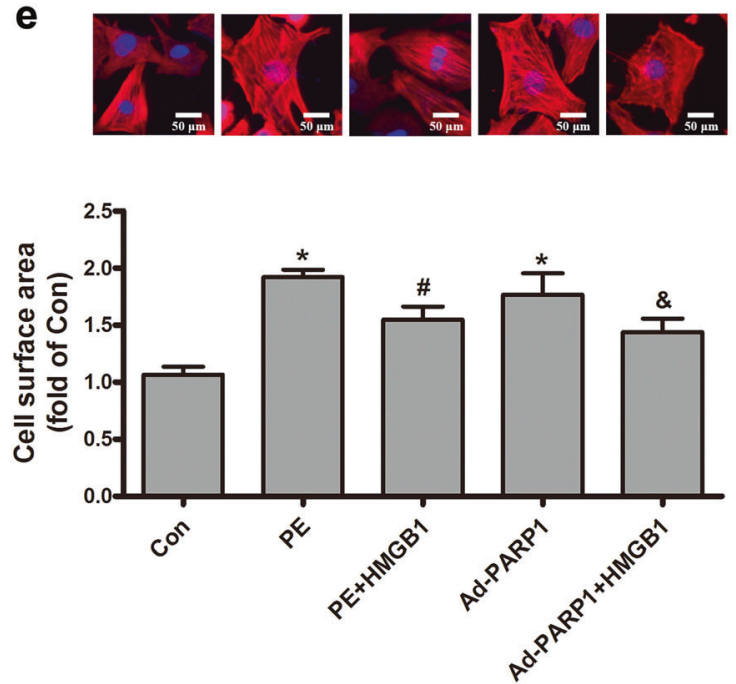

Fig. 2 The effect of PARP1 and HMGB1 on PE-induced cardiac hypertrophy. NRCMs were infected with Ad-PARP1 or Ad-GFP (empty vector), and the hypertrophic responses of NRCMs were determined by measuring a the cell surface area and $\mathbf{b}$ the mRNA expression of ANF and BNP. NRCMs were transfected with si-PARP1 or incubated with AG14361 $(1 \mu \mathrm{M})$ followed by incubation with PE $(100 \mu \mathrm{M})$. c The cell surface area was measured by dying with rhodamine-phalloidin. $\mathbf{d}$ The MRNA levels of PARP1, ANF and BNP were confirmed using real-time PCR analysis. NRCMs were transfected with HMGB1, followed by infecting with Ad-PARP1 or incubation with PE. e The cell surface was observed using rhodamine-phalloidin dying. $f$ The mRNA levels of ANF and BNP were determined by real-time PCR analysis. ${ }^{*} P<0.05$, ${ }^{* *} P<0.01$ vs control group, ${ }^{\#} P<0.05$ vs PE treatment group, ${ }^{\&} P<0.05$ vs Ad-PARP1 group, $n=5$. Con means control

for $12 \mathrm{~h}$, hinting the reduction of nucleus HMGB1 in cardiomyocyte hypertrophy. For PARP1, its total protein levels or subcellular localization was unaltered, but its catalytic activity, as determined by the PAR level, was remarkably enhanced after three stimulants treatment (Fig. 1g), which was consistent with our previous reports $[24,25]$. Upon Ang II, PE, or ISO exposure, total intracellular HMGB1 protein expression remained unchanged (Fig. 1g). Owing to HMGB1 is an exocrine protein, we tested its protein content in cell supernatant using ELISA kit, and found that its extracellular protein was obviously increased during cardiac hypertrophy (Fig. 1h).

The effect of PARP1 and HMGB1 on PE-induced cardiac hypertrophy

To explore the impact of PARP1 and HMGB1 on cardiac hypertrophy, we conducted a series of experiments. NRCMs were treated with adenovirus encoding PARP1 (Ad-PARP1) for $48 \mathrm{~h}$. The control group was incubated equal green fluorescent protein (Ad-GFP). The successful overexpression of PARP1 gene was determined by Western blot analysis (Supplemental Fig. S1). In Ad-PARP1 group, the cell surface area and the mRNA expression of ANF and BNP were prominently elevated (Fig. 2a, b), which revealed that PARP1 overexpression induced cardiac hypertrophy. The successful silencing or inhibition of PARP1 were respectively determined by Western blot analysis (Supplemental Fig. S2 and 4). By contrast, endogenic PARP1 knockdown or its specific inhibitor AG14361 both remitted PE-induced the rise of cell surface area and the upregulation of ANF and BNP mRNA (Fig. 2c, d), suggesting that reduction of PARP1 reversed PE-induced cardiac hypertrophy.

To probe the part of HMGB1 in PE-stressed myocardial hypertrophy, NRCMs were transfected with the HMGB1 plasmid with or without PE incubation. As shown in Fig. 2e, f, HMGB1 overexpression clearly alleviated hypertrophic responses by $P E$, as observed by the changes of the cell surface area and mRNA levels of ANF and BNP. Moreover, PE treatment and Ad-PARP1 infection distinctly aggravated cardiac hypertrophy according to the incremental cell surface area and the mRNA levels of ANF and BNP, while that effect was widely depressed upon HMGB1 
a

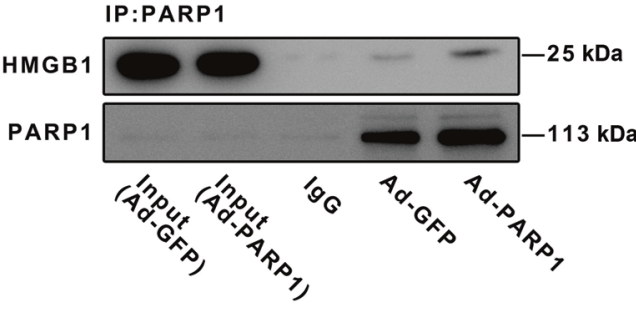

C

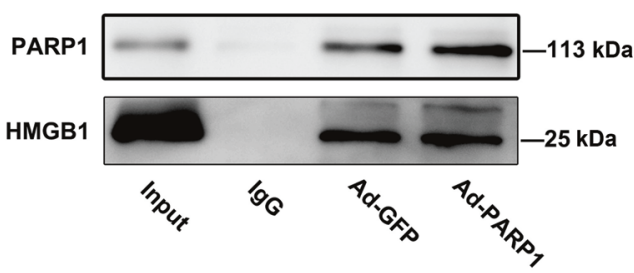

e
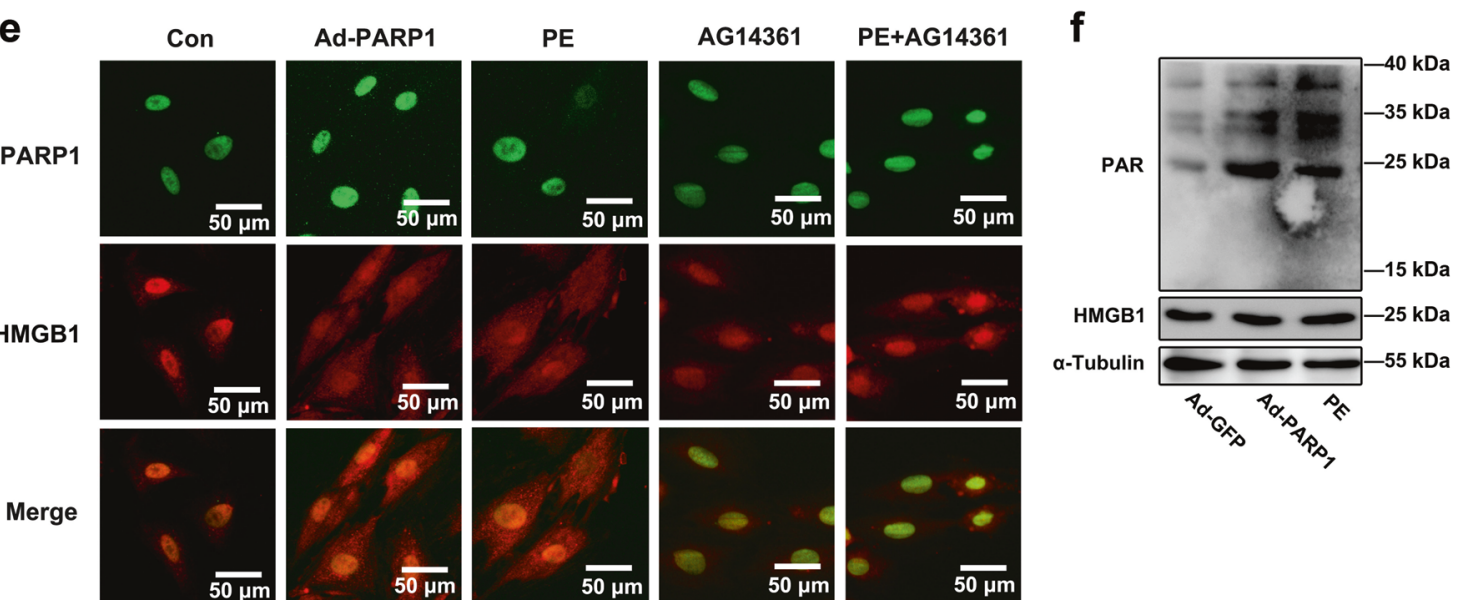

Fig. 3 The interaction between PARP1 and HMGB1 in the nucleus of NRCMs. $\mathbf{a}$, $\mathbf{b}$ NRCMs were infected with adenovirus Ad-PARP1 for $48 \mathrm{~h}$ or were incubated with PE $(100 \mu \mathrm{M})$ for $24 \mathrm{~h}$, and were precipitated by anti-PARP1, followed by subjection to co-immunoprecipitation (co-IP) assays for HMGB1 detection. c, d NRCMs were infected with Ad-PARP1 for $48 \mathrm{~h}$ or were incubated with PE (100 $\mu \mathrm{M})$ for $24 \mathrm{~h}$, and were precipitated by anti-HMGB1 for PARP1 detection in reciprocal co-IP assays. NRCMs were infected with Ad-PARP1, or were incubated with PE, followed by pre-treatment with AG14361 $(1 \mu \mathrm{M})$. e The intracellular colocalization of PARP1 and HMGB1 was confirmed in NRCMs, by immunofluorescence (IF) assay using EVOS FL Auto. Representative images of five independent experiments are shown. $\mathbf{f}$ NRCMs were infected with Ad-PARP1, or were incubated with PE, immunoprecipitated with anti-HMGB1 and subsequently subjected to immunoblotting analysis. $n=5$. Con means control

overexpression (Fig. 2e, f). Our results implied that HMGB1 was involved in PARP1-caused cardiac hypertrophy.

The interaction between PARP1 and HMGB1 in the nucleus of NRCMs

To identify whether the interaction between PARP1 and HMGB1, we first performed co-immunoprecipitation (co-IP) experiments using NRCMs. As for Fig. 3a, b, the PARP1 protein was precipitated with anti-PARP1 antibody, then Western blot analysis was performed. Under the condition of Ad-PARP1 adenovirus infection, the combination between PARP1 and HMGB1 was strengthened evidently (Fig. 3a). However, PE treatment weakened the combination between PARP1 and HMGB1 (Fig. 3b). Consistently, PARP1 was observed in precipitations gathered by the antibody against HMGB1 (Fig. 3c, d). After Ad-PARP1 infection, the interaction between PARP1 and HMGB1 was remarkably enhanced (Fig. 3c). But this interaction was reduced by $P E$ incubation (Fig. 3d). Besides, the intracellular colocalization of PARP1 and HMGB1 in NRCMs was observed by IF assay (Fig. 3e). Moreover, Ad-PARP1 increased PARylation of HMGB1, while the PARylated level was suppressed upon PE exposure (Fig. 3f). These results hinted that PARP1 interacted and PARylated HMGB1 in the nucleus of NRCMs.

The role of PARP1 in the subcellular localization and secretion of HMGB1

Since PARP1 interacted with HMGB1 in NRCMs, we further investigated the possible participation of PARP1 in regulating the subcellular localization and secretion of HMGB1. The nuclear protein of HMGB1 was investigated by determining its expression in the nucleus fractions of NRCMs and by detecting its subcellular distribution using IF assay. Our results revealed that PARP1 overexpression by infecting Ad-PARP1 promoted the nuclear export of HMGB1, facilitated the secretion of HMGB1 outside the cell (Fig. 4a, e). PARP1 silencing by transfecting with siRNA interference or its specific inhibitor AG14361 showed the opposite results (Figs. $4 b, f$ and $5 b, d, e)$. However, the overexpression, knockdown, or inhibition of PARP1 did not affect the total protein of HMGB1 (Figs. 4c, d and 5a). Besides, PE promoted the nuclear distribution of HMGB1, which could be antagonized by PARP1 silencing and AG14361 (Fig. 5d, e). Moreover, Ad-PARP1 facilitated the secretion of HMGB1 outside the cell (Fig. 5f). But the 

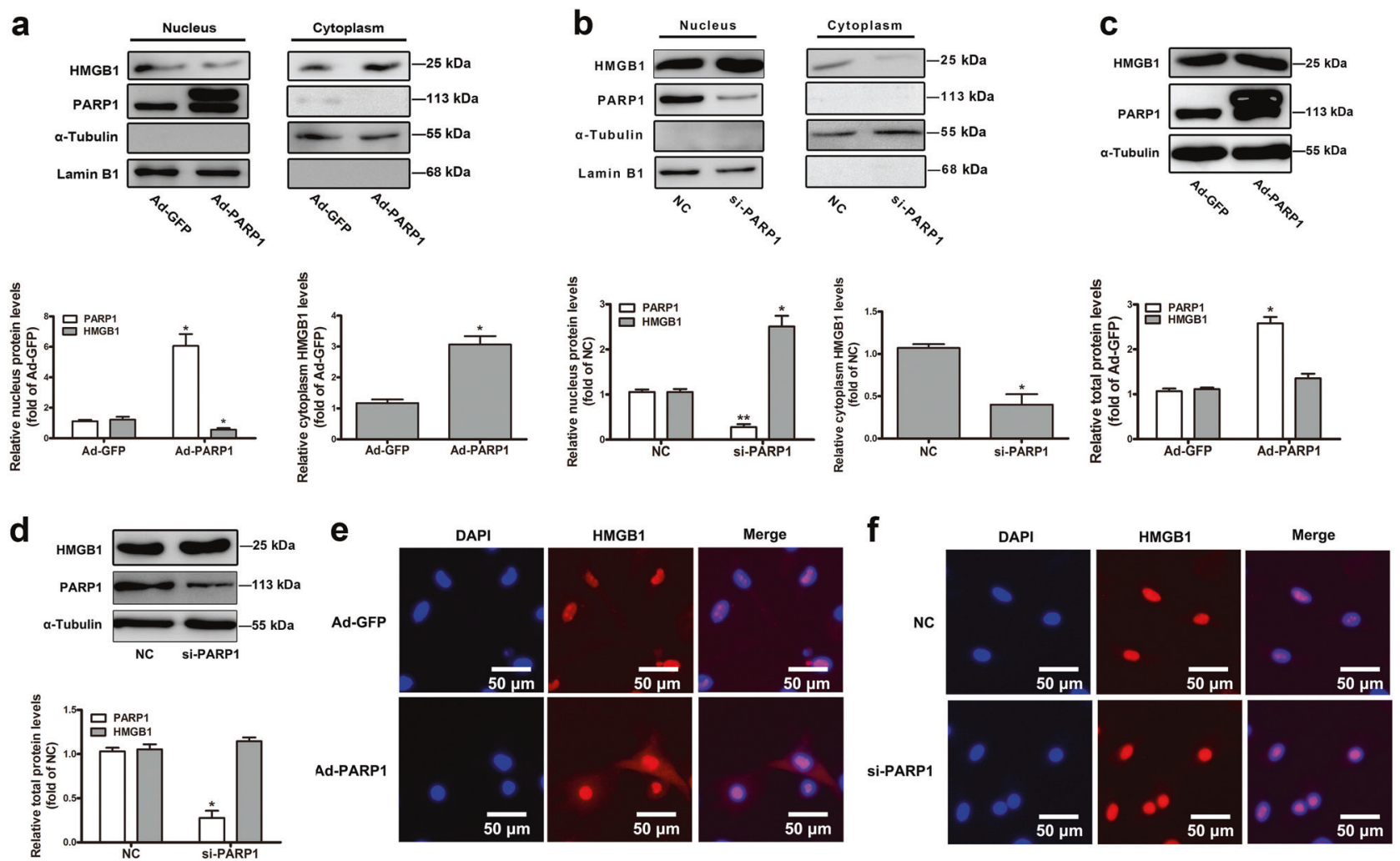

Fig. 4 The role of PARP1 in the subcellular localization of HMGB1. NRCMs were infected with Ad-PARP1 or Ad-GFP (empty vector) for 48 h. In addition, another group of NRCMs were transfected with si-PARP1 or negative control (NC) for $72 \mathrm{~h}$. $\mathbf{a}$, $\mathbf{b}$ The nuclear and cytoplasmic protein were extracted respectively from NRCMs, and were observed by Western blot analysis. c, $\mathbf{d}$ Western blot analysis was conducted to determine the intracellular proteins of HMGB1 and PARP1. The results were normalized to those of $\alpha$-Tubulin/Lamin B1 and were presented as the means \pm SEM. ${ }^{*} P<0.05$ vs Ad-GFP group or NC group, $n=5$. e, $\mathbf{f}$ Immunofluorescence (IF) assay was performed to detect the subcellular distribution of HMGB1. Representative images of five independent experiments are shown. Con means control, NC means negative control

intracellular protein of HMGB1 was obviously increased in the knockdown or inhibition of PARP1 group (Fig. 5c, g).

The inhibition of PARP1 relieved AAC-induced cardiac hypertrophy According to published reports, AAC surgery had widely been used to imitate pressure-overload-stressed cardiac hypertrophy $[24,30]$. In this study, AAC pressure overload for 6 weeks, and a series of experiments were conducted to confirm the successful establishment of cardiac hypertrophy model. The hearts of AAC group were obviously larger than those from control animals receiving sham-operation (Sham) and revealed typical hypertrophic changes, as showed by HE and Masson staining (Fig. 6a, $\mathrm{i}$-iii). Additionally, the heart weight-to-tibia length (HW/TL) ratio was enhanced upon AAC treatment (Fig. 6b). Echocardiography showed that ejection fraction (EF), fractional shortening (FS), cardiac output (CO), heart rate (HR), and left ventricular internal diameter (LVID) were reduced while the interventricular septum (IVS) and left ventricular posterior wall thickness (LVPW) were both increased in AAC-treated rats (Fig. 6a, iv, C-i). These results hint the successful induction of myocardial hypertrophy by AAC. To investigate the role of PARP1 inhibition in AAC-induced cardiac hypertrophy, 3AB (3-aminobenzamide, MCE, New Jersey, USA), a well-established PARP inhibitor $[24,25,30]$, was given $(20 \mathrm{mg} / \mathrm{kg}$ every day, ip) after AAC surgery for $7 \mathrm{~d}$ and continued for 6 weeks. The results of morphological and echocardiographic analysis displayed a tendency of mitigation in $A A C+3 A B$ group, as compared to that with AAC surgery (Fig. $6 a-i)$. In the hearts of SD rats, AAC prominently promoted the translocation of HMGB1 from nucleus to cytoplasm, which could be antagonized by $3 A B$, according to the results of IF assay (Fig. 6j).

\section{DISCUSSION}

In this report, we identified that: (1) HMGB1 was excluded from the nucleus after treatment with three cardiac hypertrophic stimulants (Ang II, PE, or ISO) for $12 \mathrm{~h}[35,36]$; (2) PARP1 interacts and PARylates HMGB1, and induces nuclear export of HMGB1 in cardiomyocytes; (3) PARP1 silencing or inhibition retarded PEcaused HMGB1 nuclear export; (4) The nuclear HMGB1 content is partially responsible for the prevention of PARP1-mediated cardiac hypertrophy. We provide novel evidence that PARP1 combines with HMGB1 and accelerates its transposition from nucleus to cytoplasm, and finally causes cardiac hypertrophy.

HMGB1 had three paralogs (proteins that have substantial sequence similarity in common) in mammals, HMGB2, 3, and 4. Yet $\mathrm{HMGB} 1^{-/-}$mice died during late gestation or within $24 \mathrm{~h}$ of birth, which clued the importance of HMGB1 [37]. HMGB1 exhibited diverse functions according to its subcellular location [6]. In the nucleus, HMGB1 acted as DNA chaperone and participated in various nuclear processes, such as transcription, replication, DNA repair and nucleosome assembly [4-7]. Besides its nuclear function, HMGB1 serves as an inflammatory cytokine when secreted from stressed cells [8-10]. When it comes to inflammation, the production of HMGB1 and its receptor TLR4 increases in LPS-induced model of inflammation [38]. Furthermore, the model of TLR4 knockout rescues against paraquat exposure-induced myocardial and sarcomere contractile dysfunction [39]. HMGB1 is abundantly expressed in most kinds of mammalian cells, including cardiomyocytes, macrophages, and immune cells $[6,10]$. In this paper, HMGB1 was predominately located in the nucleus of normal cardiomyocytes, but excluded from the nucleus upon Ang II, PE, or ISO exposure for $12 \mathrm{~h}[35,36]$. We also found the 

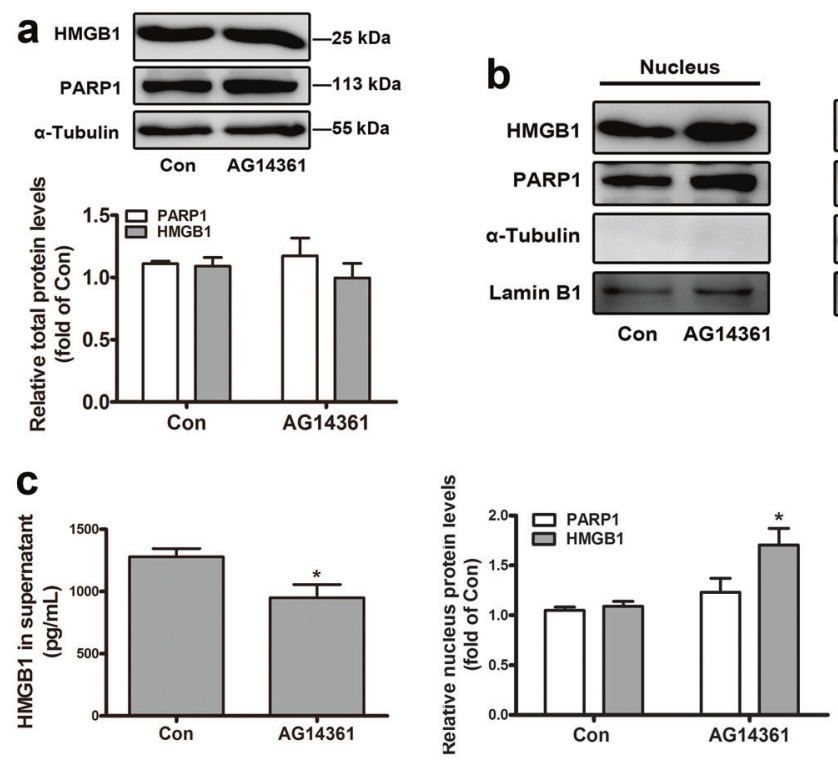

d
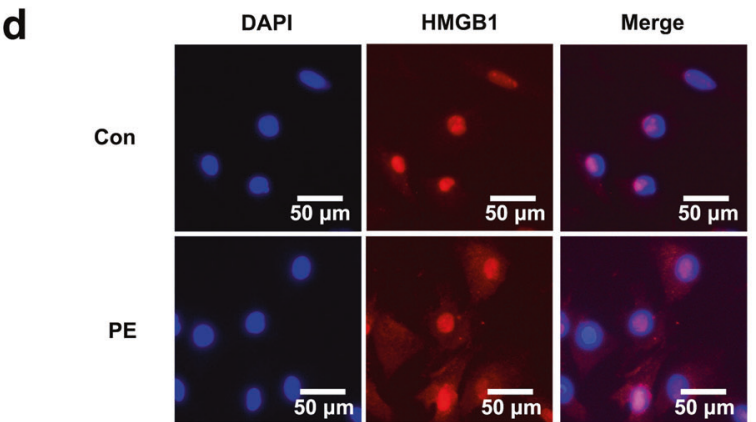

AG1436
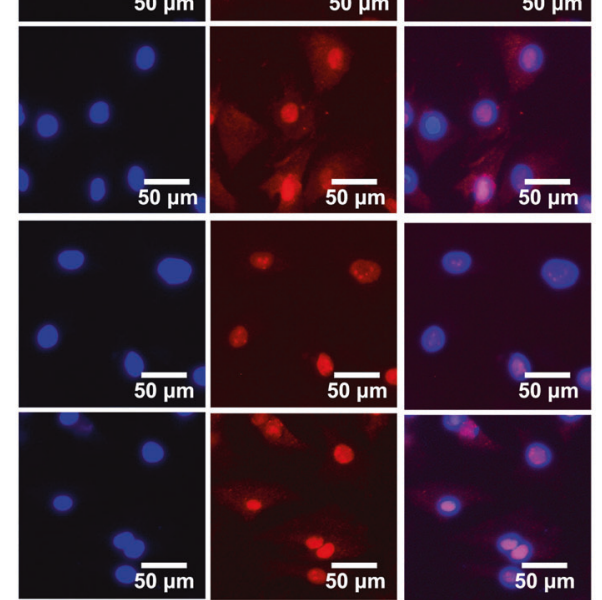

$50 \mu \mathrm{m}$
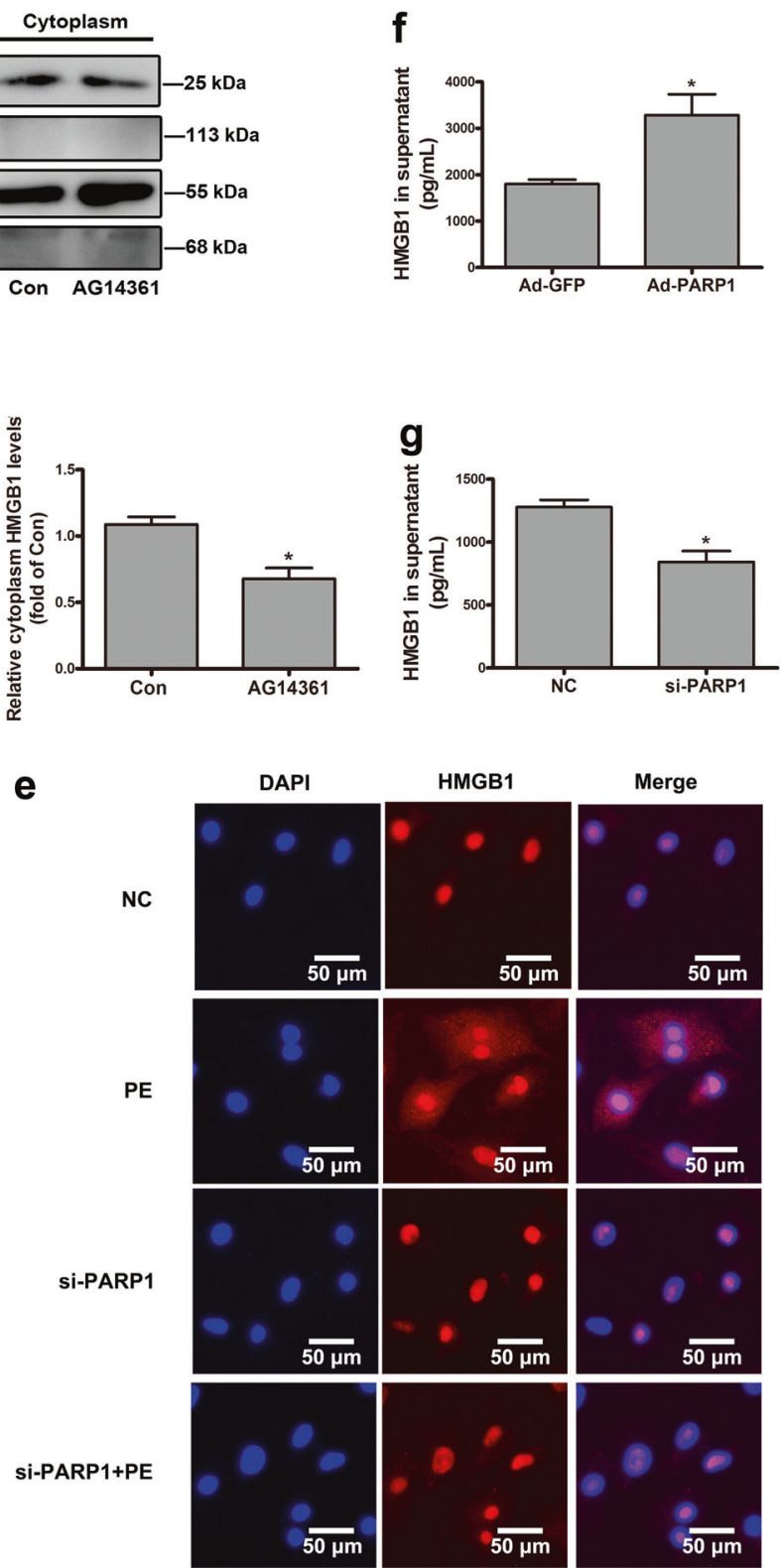

Fig. 5 The effect of PARP1 on the subcellular localization and secretion of HMGB1. NRCMs were treated with AG14361 (1 $\mu$ M, $24 \mathrm{~h})$ with or without PE exposure. Besides, the other two groups of NRCMs were transfected with Ad-PARP1 or si-PARP1. a Western blot analysis was conducted to determine the intracellular proteins of HMGB1 and PARP1. b The nuclear and cytoplasmic proteins were extracted respectively from NRCMs, and were observed by Western blot analysis. c, f, $\mathbf{g}$ ELISA experiment was operated to detect the level of HMGB1 in cell supernatant. The results were normalized to those of $\alpha$-Tubulin/Lamin B1 and were presented as the means \pm SEM. ${ }^{*} P<0.05$ vs control group, $n=5$. d, e Immunofluorescence (IF) assay was performed to detect the subcellular distribution of HMGB1. Representative images of five independent experiments are shown. Con means control, NC means negative control

increased secretion of HMGB1 in all three of hypertrophic models, despite the total intracellular HMGB1 protein expression remained unchanged.

In physiological conditions, mild PARP activation regulated many cellular courses, including gene transcription, DNA repair, and cell-cycle progression [23]. However, excessive activation of PARP was involved in multiple pathological conditions, such as circulatory shock, inflammation, stroke, and reperfusion injury of many organs $[16,40,41]$. PARP inhibition has proved effective in preventing heart remodeling and delaying the transition of hypertensive cardiopathy to HF in spontaneously hypertensive rats (SHRs) [42]. PARP1 ${ }^{-1-}$ mice are protected against Ang II- stressed cardiac hypertrophy [24]. We have covered that PARP1 is strongly activated by Ang II or ISO, while two novel inhibitors of PARP1 (salvianolic acid B and AG-690/11026014) prevent myocardium from Ang II-stressed hypertrophy in vitro and in vivo [24]. Besides, our previous research have demonstrated that NOR1 promoted cardiac hypertrophy partly owing to its regulation on PARP1 enzymic activity [33]; the PARylation of FoxO3 induced by PARP1 facilitates its phosphorylation at critical sites, leading to its translocation from the nucleus and finally resulting in cardiac hypertrophy [25]. Consistent with our previous reports, the total protein level or subcellular localization of PARP1 was unaltered, but its catalytic activity, as determined by the PAR level, was 
a

(i)

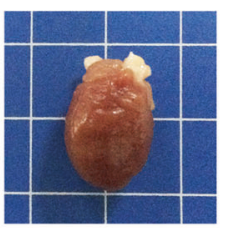

(ii)

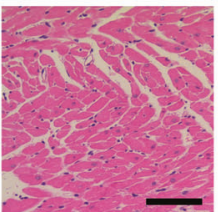

(iii)

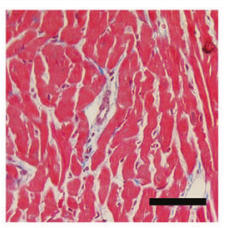

(iv)

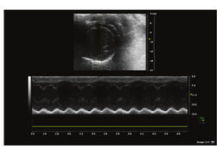

Sham
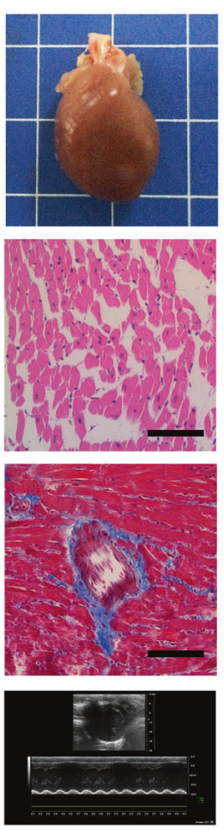

AAC
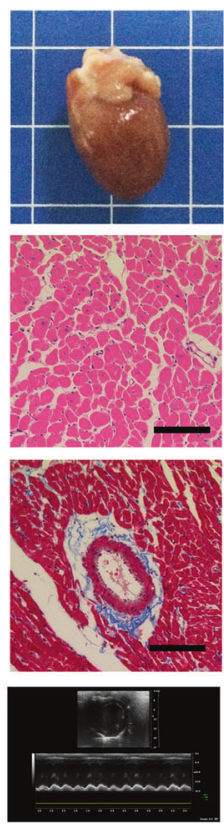

$A A C+3 A B$ b

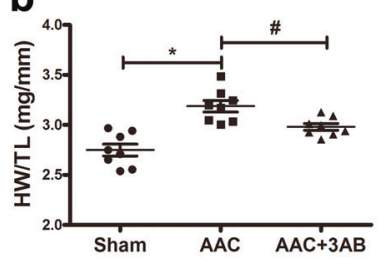

d

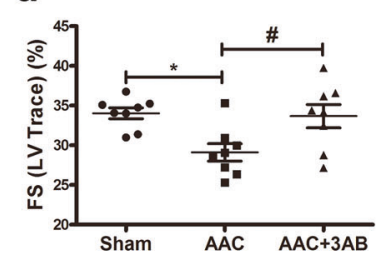

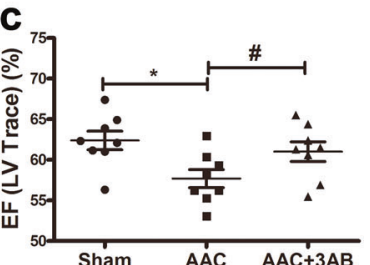

e

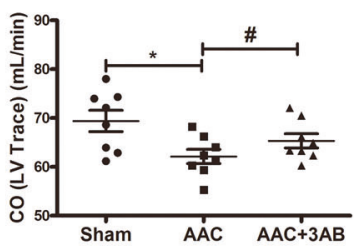

f

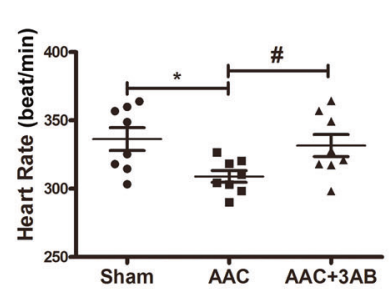

h

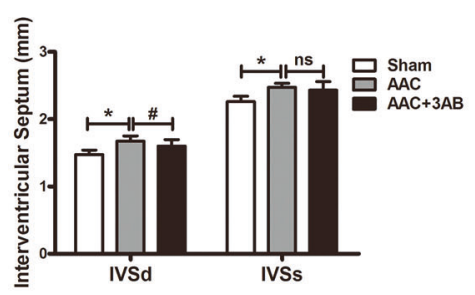

g

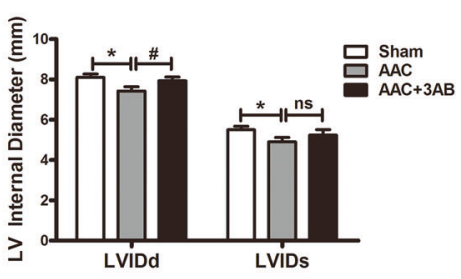

i

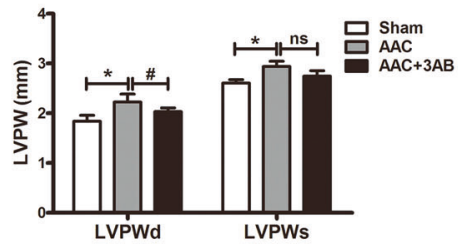

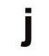

j

Sham
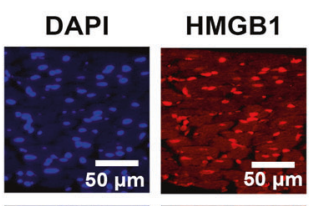

Merge

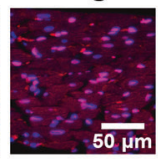

AAC
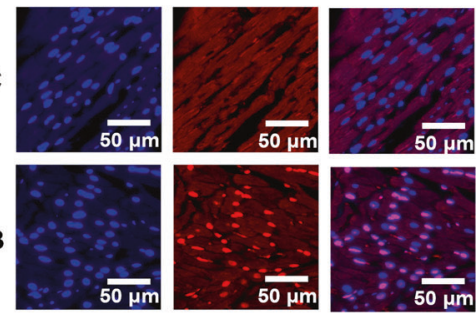

$A A C+3 A B$
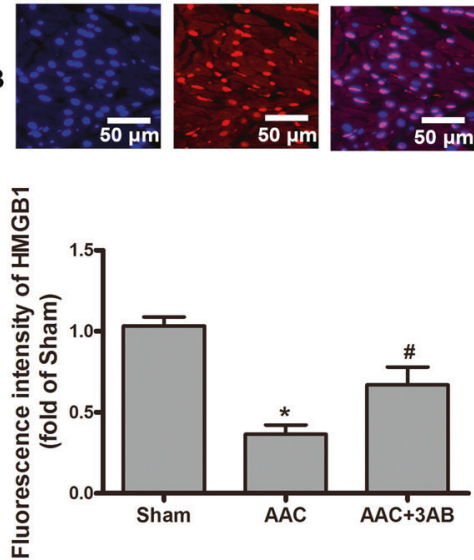

Fig. 6 The inhibition of PARP1 relieved abdominal aortic constriction (AAC)-induced cardiac hypertrophy. a Gross heart, HE, or Masson-stained cross sections of the left ventricle (magnification, $\times 200$ ), and representative echocardiographic graphs were shown. b Post-mortem measurements of heart weight/tibia length $(\mathrm{HW} / \mathrm{TL}, \mathrm{mg} / \mathrm{mm})$. c-i Echocardiography measurements of ejection fraction (EF, \%), fraction shortening (FS, \%), cardiac output (CO, mL/min), heart rate (HR, BPM), LV internal diameter (LVID, mm), interventricular septum (IVS, mm), and left ventricular posterior wall thickness (LVPW, mm). j Immunofluorescence assay was carried out to detect the subcellular localization of HMGB1 using EVOS FL Auto and the fluorescence intensity of HMGB1 in rat myocardium was measured. ${ }^{*} P<0.05$ vs Sham group. ${ }^{\#} P<0.05$ vs AAC group, $n=8$. ns means no statistical difference

remarkably enhanced after three stimulants treatment. PARP1 overexpression induced cardiac hypertrophy, as shown by the enhanced cell surface area and the mRNA expression of ANF and BNP. By contrast, endogenic PARP1 knockdown or its specific inhibitor AG14361 both reversed PE-induced cardiac hypertrophy. Similarly, the inhibition of PARP1 by its inhibitor $3 A B$ observably attenuated AAC-induced myocardial hypertrophic responses in vivo.

It had been reported in the last few years that the expression of HMGB1 was obviously less in human failing heart samples than in normal hearts because of the migration from nucleus to cytoplasm in failing hearts [4]. Exogenous HMGB1 was proven to induce cardiac hypertrophy [12]. In this work, we identified that HMGB1 overexpression clearly alleviated hypertrophic responses by PE. More intriguingly, PE treatment or Ad-PARP1 infection both distinctly aggravated cardiac hypertrophy, while that effect was widely depressed upon HMGB1 overexpression.

PARP1 was necessary for TNFSF10-induced ADP-ribosylation of HMGB1 in cancer cells. Furthermore, PARP1-mediated PARylation of HMGB1 was required for passive HMGB1 release during injury 
[43]. It had been reported that DNA damage by alkylating agents activated PARP1, accompanied by translocation of HMGB1 into the cytoplasm and subsequent released into the extracellular space due to increased permeability of the cell membrane [36]. The migration of HMGB1 from nucleus to cytoplasm was PARP1dependent, which served to establish the capacity of cells to release this valid inflammatory mediator onto subsequent necrotic death [26]. We found that PARP1 interacted with HMGB1 in the nucleus of cardiomyocytes, whereas this combination was weakened by PE-caused cardiac hypertrophy. The interaction between PARP1 and HMGB1 may promoted the nuclear distribution of HMGB1, facilitated the secretion of HMGB1 outside the cell, although did not affect the total protein of HMGB1. Similarly, PE led to the transfer from the nucleus of HMGB1, promoted intracellular HMGB1, which could be antagonized by PARP1 silencing and its inhibitor AG14361.

HMGB1 contained two HMG DNA-binding domains, called A and $B$ boxes, two nucleus localization sites (NLSs), located in the $A$ box (aa 28-44) and in the B box (aa 179-185), respectively, directed the migration of the protein into the nucleus [37]. Previous research revealed that post-translational modifications determined the fate of HMGB1 [10]. Acetylation of its NLS lysines was critical for the translocation from nucleus to cytoplasm and the release of the protein by immune cells [36]. Moreover, the nucleus-to-cytoplasm shuttling of HMGB1 was also regulated by serine/threonine phosphorylation in the two NLS [37]. Interestingly, mono-methylation of lysine42 was another modulatory mechanism of HMGB1 nucleus-to-cytoplasm shuttling [37]. In our research, we found that PARP1 interacted and PARylated HMGB1, suggesting that PARP1 played the part by the means of posttranslational modification.

Further studies are required to confirm the PARylated modification of HMGB1 by PARP1, and to explore its exact PARylation sites. Besides, HMGB1 exits from the cell via two different patterns: passive or active release [12]. We are interested in which way HMGB1 was secreted by PARP1.

\section{CONCLUSION}

In conclusion, our present study reveals that PARP1 interacts with HMGB1 and induces nuclear export of HMGB1 in cardiomyocytes, and results in cardiac hypertrophy. The nuclear HMGB1 content is partially responsible for the prevention of PARP1-mediated cardiac hypertrophy.

\section{ACKNOWLEDGEMENTS}

This research was supported by grants from the 111 Project (No. B16047 to Pei-qing LIU), National Natural Science Foundation of China (81473205, 81673433 to Pei-qing LIU), Major Project of Platform Construction Education Department of Guangdong Province (No. 2014GKPT002 to Pei-qing LIU), and Special Program for Applied Science and Technology of Guangdong Province (No. 2015B020232009, 2014B020210003 and 2013B090700010 to Pei-qing LIU), National Engineering and Technology Research Center for New Drug Druggability Evaluation (Seed Program of Guangdong Province), Guangzhou Science and Technology Program Project (No. 201604020121 to Pei-qing LIU), Medical Scientific Research Foundation of Guangdong Province (No. A2018078 to Jing LU).

\section{AUTHOR CONTRIBUTION}

ZML and PQL designed the study. QL, SYS, LPW, ZG performed the experiments. QL, PXW, and HWY analyzed the data. QL, JL, JTY wrote the manuscript.

\section{ADDITIONAL INFORMATION}

The online version of this article (https://doi.org/10.1038/s41401-018-0044-4) contains supplementary material, which is available to authorized users.

Competing interests: The authors declare no competing interest.

\section{REFERENCES}

1. Yu SS, Cai Y, Ye JT, Pi RB, Chen SR, Liu PQ, et al. Sirtuin 6 protects cardiomyocytes from hypertrophy in vitro via inhibition of NF-KB-dependent transcriptional activity. Br J Pharmacol. 2013;168:117-28.

2. Frey $\mathrm{N}$, Katus $\mathrm{HA}$, Olson EN, Hill JA. Hypertrophy of the heart: a new therapeutic target. Circulation. 2004;109:1580-9.

3. Glennon PE, Sugden PH, Poole-Wilson PA. Cellular mechanisms of cardiac hypertrophy. Br Heart J. 1995;73:496-9.

4. Funayama A, Shishido T, Netsu S, Narumi T, Kadowaki S, Takahashi H, et al. Cardiac nuclear high mobility group box 1 prevents the development of cardiac hypertrophy and heart failure. Cardiovasc Res. 2013;99:657-64.

5. Srinivasan M, Banerjee S, Palmer A, Zheng G, Chen A, Bosland MC, et al. HMGB1 in hormone-related cancer: a potential therapeutic target. Horm Cancer. 2014;5:127-39.

6. Jia ZK, Xue R, Liu GQ, Li L, Yang JJ, Pi GF, et al. HMGB1 is involved in the protective effect of the PPARa agonist fenofibrate against cardiac hypertrophy. PPAR Res. 2014;2014:541394.

7. Li YF, Li XH, Qu Y, Huang JC, Zhu TT, Zhao FY, et al. Role of HMGB1 translocation to neuronal nucleus in rat model with septic brain injury. Neurosci Lett. 2017;645:90-6

8. Takahashi K, Fukushima S, Yamahara K, Yashiro K, Shintani Y, Coppen SR, et al. Modulated inflammation by injection of high-mobility group box 1 recovers postInfarction chronically failing heart. Circulation. 2008;118:S106-114.

9. Kohno T, Anzai T, Naito K, Miyasho T, Okamoto M, Yokota H, et al. Role of highmobility group box 1 protein in post-infarction healing process and left ventricular remodelling. Cardiovasc Res. 2009;81:565-73.

10. Tang YT, Zhao X, Daniel A, Xiao XZ, Wang HC, Ulf A, et al. Regulation of posttranslational modifications of HMGB1 during immune responses. Antioxid Redox Sign. 2016;24:620-34.

11. Zhang L, Liu M, Jiang $H, Y u$ Y, Yu P, Tong R, et al. Extracellular high-mobility group box 1 mediates pressure overload-induced cardiac hypertrophy and heart failure. J Cell Mol Med. 2016;20:459-70.

12. Su FF, Shi MQ, Guo WG, Liu XT, Wang HT, Lu ZF, et al. High-mobility group box 1 induces calcineurin-mediated cell hypertrophy in neonatal rat ventricular myocytes. Mediat Inflamm. 2012;2012:805149.

13. Wacker DA, Frizzell KM, Zhang T, Kraus WL. Regulation of chromatin structure and chromatin-dependent transcription by poly(ADP-ribose) polymerase-1: possible targets for drug-based therapies. Subcell Biochem. 2007;41:45-69.

14. Wacker DA, Ruhl DD, Balagamwala EH, Hope KM, Zhang T, Kraus WL. The DNA binding and catalytic domains of poly(ADP-Ribose) polymerase 1 cooperate in the regulation of chromatin structure and transcription. Mol Cell Biol. 2007;27:7475-85.

15. Krishnakumar R, Kraus WL. PARP-1 regulates chromatin structure and transcription through a KDM5B-dependent pathway. Mol Cell. 2010;39:736-49.

16. Gerace E, Masi A, Resta F, Felici R, Landucci E, Mello T, et al. PARP-1 activation causes neuronal death in the hippocampal CA1 region by increasing the expression of $\mathrm{Ca}^{2+}$-permeable AMPA receptors. Neurobiol Dis. 2014;70:43-52.

17. Kanai M, Hanashiro $K$, Kim SH, Hanai S, Boulares AH, Miwa M, et al. Inhibition of Crm1-p53 interaction and nuclear export of p53 by poly(ADP-ribosyl)ation. Nat Cell Biol. 2007;9:1175-83.

18. Olabisi OA, Soto-Nieves N, Nieves E, Yang T, Yang X, Yu RY, et al. Regulation of transcription factor NFAT by ADP-ribosylation. Mol Cell Biol. 2008;28:2860-71.

19. Lönn P, van der Heide LP, Dahl M, Hellman U, Heldin CH, Moustakas A. PARP-1 attenuates Smad-mediated transcription. Mol Cell. 2010;40:521-32.

20. Sakamaki J, Daitoku H, Yoshimochi K, Miwa M, Fukamizu A. Regulation of FOXO1mediated transcription and cell proliferation by PARP-1. Biochem Bioph Res Commun. 2009;382:497-502.

21. Luo X, Nie J, Wang S, Chen Z, Chen W, Li D, et al. Poly(ADP-ribosyl) ation of FOXP3 protein mediated by PARP-1 protein regulates the function of regulatory $T$ cells. J Biol Chem. 2015;290:28675-82.

22. Ju BG, Solum D, Song EJ, Lee KJ, Rose DW, Glass CK, et al. Activating the PARP-1 sensor component of the groucho/TLE1 corepressor complex mediates a CaMKinase Ildelta-dependent neurogenic gene activation pathway. Cell. 2004;119:815-29.

23. Pillai JB, Gupta M, Rajamohan SB, Lang R, Raman J, Gupta MP. Poly(ADP-ribose) polymerase-1-deficient mice are protected from angiotensin Il-induced cardiac hypertrophy. Am J Physiol Heart Circ Physiol. 2006;291:H1545-H1553.

24. Feng GS, Zhu CG, Li ZM, Wang PX, Huang $Y$, Liu $M$, et al. Synthesis of the novel PARP-1 inhibitor AG-690/11026014 and its protective effects on angiotensin II-induced mouse cardiac remodeling. Acta Pharmacol Sin. 2017;38:638-50.

25. Lu J, Zhang RW, Hong HQ, Yang ZL, Sun DP, Sun SY, et al. The poly(ADP-ribosyl) ation of FoxO3 mediated by PARP1 participates in isoproterenol-induced cardiac hypertrophy. Biochim Biophys Acta. 2016;1863:3027-39. 
26. Ditsworth D, Zong WX, Thompson CB. Activation of poly(ADP)-ribose polymerase (PARP-1) induces release of the pro-inflammatory mediator HMGB1 from the nucleus. J Biol Chem. 2007;282:17845-54.

27. Lu J, Sun DP, Liu ZP, Li M, Hong HQ, Liu C, et al. SIRT6 suppresses isoproterenolinduced cardiac hypertrophy through activation of autophagy. Transl Res. 2016;172:96-112.e6.

28. Yue ZB, Ma YZ, You J, Li ZM, Ding YQ, He P, et al. NMNAT3 is involved in the protective effect of SIRT3 in Ang II-induced cardiachypertrophy. Exp Cell Res. 2016;347:261-73.

29. Shen PY, Feng XJ, Zhang XY, Huang XY, Liu SL, Lu X, et al. SIRT6 suppresses phenylephrine-induced cardiomyocyte hypertrophy though inhibiting p300. J Pharmacol Sci. 2016;132:31-40.

30. Sun SY, Hu YH, Zheng QY, Guo Z, Sun DP, Chen SR, et al. PARP1 induces cardiac fibrosis by mediating mTOR activity. J Cell Biochem. 2017. https://doi.org/ 10.1002/jcb.26649.

31. Gravning J, Ahmed MS, von Lueder TG, Edvardsen T, Attramadal H. CCN2/CTGF attenuates myocardial hypertrophy and cardiac dysfunction upon chronic pressure-overload. Int J Cardiol. 2013;168:2049-56.

32. Zhang XY, Li W, Shen PY, Feng XJ, Yue ZB, Lu J, et al. STAT3 suppression is involved in the protective effect of SIRT6 against cardiomyocyte hypertrophy. J Cardiovasc Pharmacol. 2016;68:204-14.

33. Feng XJ, Gao H, Gao S, Li ZM, Li H, Lu J, et al. The orphan receptor NOR1 participates in isoprenaline-induced cardiac hypertrophy by regulating PARP-1. Br J Pharmacol. 2015;172:2852-63.

34. Putinski C, Abdul-Ghani $M$, Stiles $R$, Brunette $S$, Dick SA, Fernando $P$, et al. Intrinsic-mediated caspase activation is essential for cardiomyocyte hypertrophy. Proc Natl Acad Sci U S A. 2013;110:E4079-4087.
35. Wu CX, He LX, Guo H, Tian XX, Liu Q, Sun H. Inhibition effect of glycyrrhizen in lipopolysaccharide-induced high-mobility group box 1 releasing and expression from RAW264.7 cells. Shock. 2015;43:412-21.

36. Yang ZY, Li L, Chen L, Yuan WW, Dong LM, Zhang YS, et al. PARP-1 mediates LPSinduced HMGB1 release by macrophages through regulation of HMGB1 acetylation. J Immunol. 2014;193:6114-23.

37. Venereau E, De Leo F, Mezzapelle R, Careccia G, Musco G, Bianchi ME. HMGB1 as biomarker and drug target. Pharmacol Res. 2016;111:534-44.

38. Ren J, Xu XH, Wang QR, Ren SY, Dong ML, Zhang YM. Permissive role of AMPK and autophagy in adiponectin deficiency-accentuated myocardial injury and inflammation in endotoxemia. J Mol Cell Cardiol. 2016;93:18-31.

39. Lei YH, Li X, Yuan F, Liu L, Zhang J, Yang YP, et al. Toll-like receptor 4 ablation rescues against paraquat-triggered myocardial dysfunction: role of ER stress and apoptosis. Environ Toxicol. 2017;32:656-68.

40. Yu C, Kim BS, Kim E. FAF1 mediates regulated necrosis through PARP1 activation upon oxidative stress leading to dopaminergic neurodegeneration. Cell Death Differ. 2016;23:1873-85.

41. Andrabi SA, Umanah GK, Chang C, Stevens DA, Karuppagounder SS, Gagné JP, et al. Poly (ADP-ribose) polymerase-dependent energy depletion occurs through inhibition of glycolysis. Proc Natl Acad Sci U S A. 2014; 111:10209-14.

42. Bartha E, Solti I, Kereskai L, Lantos J, Plozer E, Magyar K, et al. PARP inhibition delays transition of hypertensive cardiopathy to heart failure in spontaneously hypertensive rats. Cardiovasc Res. 2009;83:501-10.

43. Yang $M H$, Liu LY, Xie M, Sun XF, Yu Y, Kang R, et al. Poly-ADP-ribosylation of HMGB1 regulates TNFSF10/TRAIL resistance through autophagy. Autophagy. 2015;11:214-24. 